\title{
DISTRIBUTION ET ABONDANCE DE SPORES DE CHAMPIGNONS ENDOMYCORHIZOGENES A ARBUSCULES DANS DIFFERENTS TYPES DE FORETS DE LA TENE EN CÔTE D'IVOIRE
}

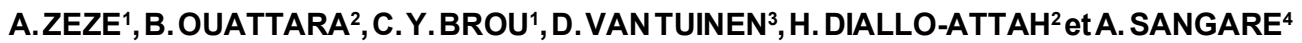 \\ ${ }^{1}$ Laboratoire d'Agronomie, Ecole Supérieure d'Agronomie, Institut National Polytechnique, BP 1313 Yamoussoukro, \\ Côte d'Ivoire. Email : zomure@yahoo.com \\ Université Abobo-Adjamé, UFR Sciences Naturelles, 02 BP 801 Abidjan 02. Côte d'Ivoire. \\ ${ }^{3}$ Unité Mixte de Recherche INRA/Université de Bourgogne, Biochimie, Biologie Cellulaire et Ecologie des Interactions \\ Plantes/Microorganismes, CMSE-INRA, BP 86510, 21065 Dijon Cédex, France \\ ${ }^{4}$ Laboratoire de biotechnologies, Centre National de la Recherche Agronomique.
}

\begin{abstract}
RESUME
L'importance des champignons endomycorhizogènes à arbuscules en agriculture durable n'est plus à démontrer. En vue d'obtenir une collection de ces champignons en Côte d'Ivoire, une prospection a été effectuée dans la forêt de la Téné (Centre-Ouest Côte d'lvoire). Cette étude a révélé la présence de ces champignons dans les différentes forêts étudiées. L'amplification en réaction de polymérisation en chaîne (PCR) des racines échantillonnées et le comptage directe des spores des sols échantillonnés ont permis de montrer que les forêts reboisées contiennent plus de champignons mycorhiziens. L'analyse par PCR des racines de niébé inoculées par différents sols montre que cette légumineuse est un bon hôte pour ces champignons et permet ainsi de les conserver au laboratoire.
\end{abstract}

Mots clés : Champignons endomycorhizogènes à arbuscules, abondance, forêts de la Téné, Côte d'ivoire.

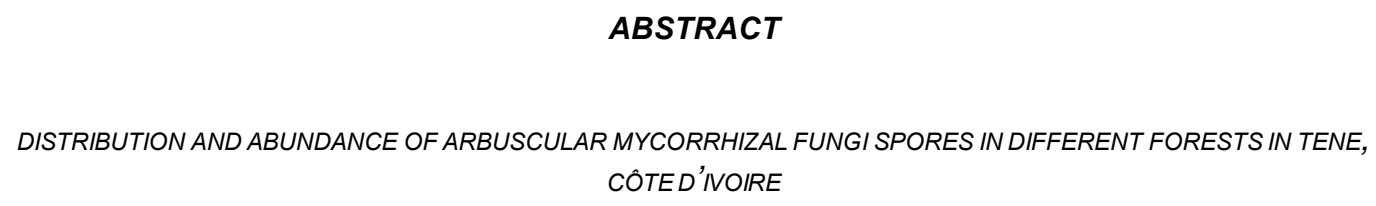

Arbuscular mycorrhizal fungi play a key role in sustainable agriculture. In order to establish a bank for the species, the fungi were isolated from soils under different land use types in Téné Forest, Center-west Côte d'lvoire. Results rshow the presence of spores in the different land use types. Amplification of the polymerisation reaction (PCR)chain in roots and direct spore counts in sampled soils show that reforested areas exhibited more arbuscular mycorrhizal fungi root colonisation. Analysis of inoculated roots from cowpea, sing the PCR technique, reveal that this plant was an efficient host for arbuscular mycorrhizal fungi conservation under laboratory conditions.

Keywords : Arbuscular mycorrhizal fungi, spore counts, Téné forests, Côte d'lvoire. 


\section{INTRODUCTION}

En milieu tropical humide, l'extension incontrôlée de l'agriculture de rente, l'exploitation industrielle du bois et l'agriculture itinérante sur brûlis font l'objet d'une préoccupation mondiale parce qu'elles causent la dégradation des forêts tropicales et même leur disparition. La déforestation galopante peut être à l'origine de la perte de diversité biologique et fonctionnelle (Onguene, 2000).

La fertilité et la productivité des sols tropicaux sont hautement liées à l'activité biologique du sol, d'autant plus que les fertilisants sont rarement utilisés par la plus grande partie des paysans (Onguene, 2000). Le développement des plantes dépend des interactions qu'elles entretiennent avec le milieu environnemental, notamment avec les microorganismes du sol. C'est le cas des mycorhizes qui sont des symbiotes des racines des plantes. Ces champignons telluriques sont dits mycorhizogènes (Smith et Read, 1997). Ces champignons qui ont été classés récemment dans l'embranchement des Gloméromycètes (Schüssler et al., 2001) constituent une composante importante dans le fonctionnement et la diversité des écosystèmes terrestres par leur ubiquité et leur implication directe dans les processus essentiels se tenant à l'interface sol/ plante (Fitter, 1988 ; Smith et al., 1993, Varma, 1998). Parmi ces champignons symbiotiques, les champignons endomycorhiziens à arbuscules et vésicules constituent le groupe le plus couramment rencontré dont les effets bénéfiques sur la croissance et la tolérance aux stress de la majorité des plantes économiquement importants sont admis (Barea et al., 1993 ; Bethlenfalvay et Lindermann, 1992 ; Smith et Read, 1997).

L'utilisation de ces champignons en agriculture durable est une réalité au niveau mondial. Cette approche nécessite la compréhension de la diversité et de la dynamique de ces champignons dans leur environnement naturel.

La forêt de la Téné, située à Oumé (Centre-Ouest de la Côte-d'Ivoire), patrimoine forestier de la Société de Devéloppement des Forêts (SODEFOR), a été choisie en vue d'apprécier la distribution des communautés naturelles des mycorhizes à arbuscules et vésicules. La zone d'étude est composée d'une forêt naturelle et d'une forêt issue de reboisement.
Les objectifs de ce travail sont de mettre en évidence la présence de champignons mycorhiziens à arbuscules dans la forêt de la Téné et de déterminer l'effet du type de forêt sur l'abondance des champignons mycorhiziens à arbuscules.

\section{MATERIEL ET METHODES}

\section{DESCRIPTION DE LA ZONE D’ETUDE}

La zone d'étude est située dans la région d'Oumé, au Centre-Ouest de la Côte d'Ivoire. C'est une zone à climat tropical humide, essentiellement forestière. Le socle est en grande partie constitué de granite. Le sol, de type ferrugineux tropical a une texture sableuse et présente un horizon gravillonnaire. La moyenne annuelle pluviométrique est de 1500 m (Avenard et al., 1971). La forêt d'Oumé est dense et semi-décidue. Elle est composée de 2 parties : la forêt naturelle et la forêt issue de reboisement.

La forêt naturelle (FN) : elle est divisée en deux sous-zones que sont la forêt primaire (FP) (semidécidue d'une densité de 41 à $60 \%$ et la forêt secondaire (FS). II s'agit d'une forêt semidécidue qui a subi les ravages d'un feu de brousse en 1983.

la forêt issue de reboisement (FR) : elle représente la zone de reboisement de la SODEFOR. Elle est subdivisée en 3 sous-zones que sont :

- le reboisement plurispécifique (RPS) effectué en 1981 avec les espèces Gmelina arborea, Terminalia superba (Fraké), et Tectona grandis (Teck)

- les reboisements monospécifiques de teck de 1994 (TK.94) et de 2000 (TKoo)

\section{PRELEVEMENT DES ECHANTILLONS DE SOLET DE RACINES}

Une grille d'échantillonnage a été installée dans la zone d'étude. Des points séparés de $200 \mathrm{~m}$ ont été identifiés dans chaque type de forêt. Cinq points ont été étudiés en forêt primaire. $\mathrm{Ce}$ sont : FP 13, FP 16, FP 24, FP 25 et FP 26.

En forêt secondaire (FS), 5 points ont été étudiés. Ce sont : FS 36, FS 38, FS 58, FS 59 et FS 60.

Dans la forêt issue de reboisement (FR), 15 points ont été prélevés dont 5 (RPS 42, RPS 
52, RPS 53, RPS 75 et RPS 86) en reboisement plurispécifique effectué en 1981 avec les espèces Gmelina arborea, Terminalia superba (Fraké), et Tectona grandis (Teck), 5 (TKM 20, TKM 21, TKM 31, TKM 40 et TKM 41) dans la plantation forestière industrielle de teck mise en place en 1994 (TK 94) et 5 (TKoo 80, TKoo 81, TKoo 91, TKoo 92 et TKoo 93) dans le reboisement monospécifique de teck de 2000 (TKoo).

Autour de chaque point de la grille d'échantillonnage, sont définis 12 sous-points à partir desquels sont effectués les prélèvements. Pour le prélèvement des échantillons de sol, des tarières de $10 \mathrm{~cm}$ de diamètre, des ciseaux palmistes, des désinfectants (hypochlorite de sodium, alcool $95 \%$ ) et des mètres rubans ont été utilisés. Les 12 sous échantillons bien mélangés sont conservés dans un sachet en plastique.

L'opération est répétée selon la même méthode pour tous les points de la grille. Cependant, avant de passer d'un point à un autre, la tarière est nettoyée à l'alcool $95 \%$ flambée puis, désinfectée avec de l'hypochlorite de sodium.

\section{DENOMBREMENT DIRECT DES SPORES}

Les sols prélevés sont apportés au laboratoire. En vue de mettre en évidence les spores présentes dans chaque échantillon de sol, la méthode de tamisage humide de Gerdemann et Nicolson (1963) a été utilisée. Elle s'effectue directement sur les échantillons de sol prélevés sur le terrain. Une quantité de $50 \mathrm{~g}$ de chaque échantillon de sol est mise dans $500 \mathrm{ml}$ d'eau de robinet. Le mélange est agité longuement pour l'homogénéisation, puis laissé au repos pendant $1 \mathrm{mn}$. Ensuite, il est passé à travers une série de tamis de maille $1 \mathrm{~mm}, 500 \mu \mathrm{m}$, $250 \mu \mathrm{m}, 125 \mu \mathrm{m}, 90 \mu \mathrm{m}$ et $45 \mu \mathrm{m}$, disposés respectivement l'un au-dessus de l'autre dans l'ordre ci-dessus mentionné. Les suspensions des 4 derniers tamis sont transférées chacune, dans un bécher. Le contenu de chaque bécher est observé, à la loupe binoculaire, par prélèvements successifs de petites quantités. Ces aliquotes prélevés sont renversés sur du papier mouchoir placé dans une boite de Pétri puis, observés à l'aide d'une loupe binoculaire. Les spores prélevées à l'aide d'une pince, sont mises dans des tubes à vis contenant de l'eau distillée stérile et conservées dans un réfrigérateur. Dans chaque tube sont mises uniquement les spores prélevées à un point. Cette extraction et énumération directe est répétée 3 fois avec la même quantité de sol
(50 g) pour chaque point échantillonné. Le nombre total de spores obtenues par cette méthode est désigné par $\mathrm{Mi}$.

\section{PRODUCTION DE SPORES PAR INOCULATION DE NIEBE}

Dans les sols directement échantillonnés, toutes les spores ne sont pas toujours représentées. II est donc nécessaire d'utiliser ces sols comme inoculum en vue de permettre l'éclosion éventuelle de toutes les spores. Le niébé [Vigna unguiculata (L). Walp.] de la variété TN 8863 écotype nigérien à port rampant dont le cycle dure 65 à 70 jours (Cissé et al., 1995) a été choisi comme plante-hôte. Des graines de niébé ayant un taux de germination égal à $100 \%$, sont semées dans des pots en plastique contenant $500 \mathrm{~g}$ de sol prélevés dans chaque échantillon ( 3 graines par pot et 3 pots par échantillon de sol). Les pots sont conservés dans une chambre de culture où les plantes sont exposées à la lumière de lampes électriques. Les plantes sont arrosées tous les 3 jours. Aucun amendement n'est apporté aux plantes. Après 60 jours de culture, la présence des champignons dans les racines est confirmée par la méthode de Trouvelot et al. (1987). cinquante grammes de sols sont utilisés pour extraire les spores toujours selon la méthode de tamisage humide de Gerdemann et Nicolson (1963), comme décrit précédemment. Deux témoins sont effectués dans des pots contenant du terreau synthétique et du sable dans les proportions $2 / 1$. Au préalable, le mélange terreau/sable est stérilisé à l'étuve à $121^{\circ} \mathrm{C}$ pendant $15 \mathrm{mn}$.

\section{AMPLIFICATION PCR D'ADN EXTRAIT DE RACINES}

Les champignons mycorhizogènes à arbuscules peuvent être présents sous forme d'hyphes et de spores dans le sol et sous forme d'arbuscules et de vésicules au sein des racines. Une amplification PCR utilisant des amorces spécifiques a été effectuée sur ADN extrait de racines pour rechercher ces champignons.

L'ADN de racines mycorhizées est extrait selon la méthode de Rogers et Bendich (1985). Deux types de racines ont été utilisés pour extraire l'ADN :

- les racines issues directement du site d'échantillonnage : pour chaque type de forêt, des racines contenues dans le mélange des sols de tous les points échantillonnés ont été 
récupérées pour l'extraction de l'ADN selon la méthode citée plus haut ;

- les racines de niébé inoculées en laboratoire.

Une portion de la petite sous-unité $18 \mathrm{~S}$ est amplifiée en utilisant la TaqPlus precision (Promega). Une amorce universelle d'eucaryote NS31 (Simon et al., 1992) et une amorce amplifiant spécifiquement les gloméromycètes AM1 (Helgason et al., 1999) ont été utilisées selon ces auteurs.

\section{DETERMINATION DE L'ABONDANCE DES SPORES}

La présence des spores est la méthode usuelle d'estimation de la présence des espèces de Champignons endomycorhizogènes à arbuscules. Les communautés de ces champignons présentes dans un sol peuvent être estimées en terme de nombre d'espèces présentes et d'abondance de chacune d'elles dans la communauté. L'estimation de l'abondance peut être faite par l'observation directe du nombre de spores présentes dans le sol (Gerdemann et Nicolson, 1963 ; Brundrett et al., 1994).

Deux techniques d'extraction ont été utilisées : l'extraction et l'énumération directe des spores (Mi) et l'extraction des spores par piégeage (Mî).

Les résultats sur l'abondance et la densité des spores dans les échantillons de sol seront exprimés à partir des formules ci-dessous :

Abondance $=(\mathrm{Mi}+\mathrm{Mî}) / 2$ (spores)

Densité $=\frac{\text { Abondance }}{\text { Quantité de sol }} \quad$ (spores/g de sol)

\section{ANALYSES STATISTIQUES}

Les analyses statistiques ont été effectuées à l'aide du logiciel STATISTICA 5.5. Le niveau de signification est égal à $5 \%$.

Le test de Hartiey a permis de tester l'effet du type d'occupation du sol sur l'abondance des spores, l'effet du type de forêt sur l'abondance des spores, l'effet de l'âge des zones reboisées sur l'abondance des spores et l'effet du type de reboisement sur l'abondance des spores.

\section{RESULTATS}

\section{MISE EN EVIDENCE DE MYCORHIZES DANS LES RACINES PRELEVEES AU CHAMP}

Après amplification PCR d'ADN de racines prélevées dans chaque type de forêt, seul l'ADN extrait de racines en forêt reboisée a donné un fragment (Figure 1). Aucune amplification n'a été obtenue avec les racines issues des autres forêts. Cependant l'amplification PCR des ADN extraits de racines de niébé inoculées avec les sols issus de différentes forêts, met en évidence la présence de champignons mycorhiziens en abondance dans toutes les forêts (Figure 2).

\section{MISE EN EVIDENCE DE SPORES DANS DIFFERENTES FORETS}

Les résultats montrent que les différentes forêts contiennent des spores de tailles variables (Tableau 1), que ce soit dans les sols prélevés directement ou obtenus après inoculation du niébé. Les pourcentages obtenus pour les différentes classes de taille sont les suivants : $500-250 \mu \mathrm{m}: 1 \%$ du total, $250-125 \mu \mathrm{m} 21 \%$, $125-90 \mu \mathrm{m} 56 \%$ et $90-45 \mu \mathrm{m} 22 \%$. Les spores extraites sont majoritairement de petite taille, avec $78 \%$ ayant une taille comprise entre 125 et $45 \mu \mathrm{m}$. La taille est un indice de classification des spores. Les grandes tailles sont généralement des Gigaspora sp. alors que les petites sont issues de Glomus sp. Par ailleurs une diversité de couleur a permis de distinguer 10 types de spores dans chaque type de forêt.

\section{EFFET DU TYPE DE FORET SUR L'ABONDANCE ET LADENSITE DES SPORES}

La densité des spores est moins importante dans les forêts naturelles que dans les forêts issues de reboisement. La densité croît dans 
l'ordre suivant : FP $(0,153$ spore/g de sol), FS $(0,164$ spore/g de sol), RPS $(0,217$ spore/g de sol), TKoo (0,314 spore/g de sol), Tk94 (0,380 spore/g de sol) (Tableau 2). La densité moyenne des spores en forêt naturelle $(0,16 \mathrm{spore} / \mathrm{g}$ de sol) est inférieure à la densité moyenne des spores en zone reboisée ( 0,30 spore/g de sol). La moyenne est de 0,25 spore/g de sol dans toute la zone d'étude (forêt naturelle + zone reboisée).

La figure 3 montre que les abondances ne sont pas les mêmes d'une zone à une autre. Les résultats montrent que le type de forêt a un effet très hautement significatif sur l'abondance des spores $(F=9529,134$ et $P=0,00)$.

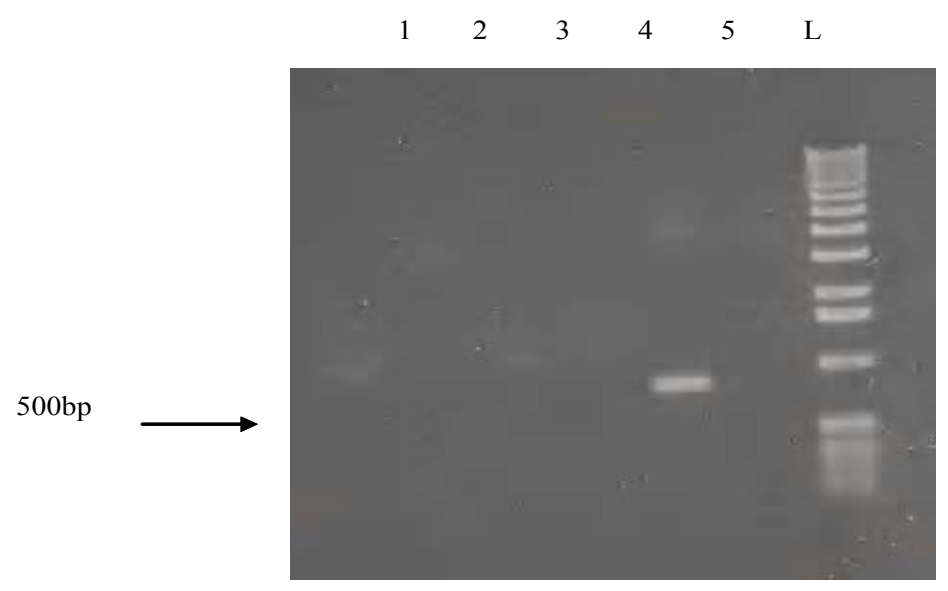

Figure 1 : Amplification PCR utilisant les amorces NS31et AM1 d'ADN de racines prélevées en 2) forêt primaire, 3) forêt secondaire, 4) forêts reboisées. Les lignes 1 et 5 représentent respectivement l'ADN 1) de niébé non mycorhizé, et 5) le contrôle sans ADN. La ligne $L$ est le ladder $500 b p$

PCR amplification using primers NS31 and AM1 of DNA from roots sampled in 2) primary forest, 3) secondary forest, 4) reafforested areas. Lines 1 and 5 represent DNA from nonmycorrhizal Vigna unguiculata and negative control respectively. Line $L$ is the $500 \mathrm{bp}$ ladder.

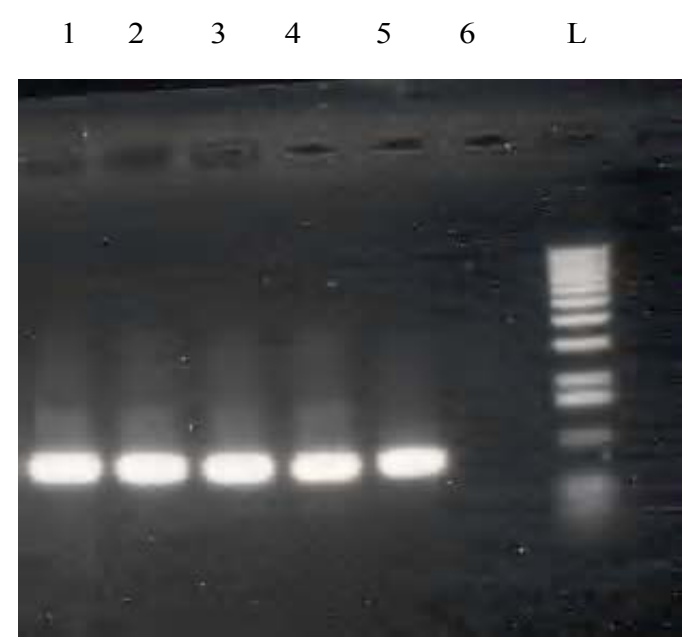

Figure 2 : Profil électonphorétique de l'amplification PCR utilisant les amorces NS31 et AM1 d'ADN de racines de niébé inoculées avec du sol issu de 1) forêt primaire, 2) forêt secondaire, 3) forêt reboisée. multispécifique, 4) forêt reboisée en 1994, 5) forêt reboisée en 2000. La ligne 6 est le contrôle négatif et $L$ le ladder $500 \mathrm{bp}$.

PCR amplification using primers NS31 and AM1 of DNA from Vigna unguiculata inoculated with soils sampled in 1) primary forest, 2) secondary forest, 3)multispecific reafforested areas, 4) reafforested areas (1994), 5) reafforested areas (2000). Line 6 represents the negative control and line $L$ the 500bp ladder. 
Tableau 1 : Dénombrement des spores en fonction de la taille $(\mu \mathrm{m})$, du type de forêt et des méthodes utilisées.

Spore count according to size $(\mu \mathrm{m})$, forest type and methods used.

\begin{tabular}{lccccccccccc}
\hline \multirow{2}{*}{$\begin{array}{l}\text { Taille } \\
\text { des spores }(\mu \mathrm{m})\end{array}$} & \multicolumn{2}{c}{$\mathrm{FP}$} & \multicolumn{2}{c}{$\mathrm{FS}$} & \multicolumn{2}{c}{$\mathrm{RPS}$} & \multicolumn{2}{c}{$\mathrm{TK}_{94}$} & \multicolumn{2}{c}{ TKoo } & \multirow{2}{*}{ Total } \\
\cline { 2 - 10 } & $\mathrm{M}_{1}$ & $\mathrm{M}_{2}$ & $\mathrm{M}_{1}$ & $\mathrm{M}_{2}$ & $\mathrm{M}_{1}$ & $\mathrm{M}_{2}$ & $\mathrm{M}_{1}$ & $\mathrm{M}_{2}$ & $\mathrm{M}_{1}$ & $\mathrm{M}_{2}$ & \\
\hline ]500;250] & 6 & 3 & 0 & 7 & 2 & 2 & 7 & 1 & 3 & 3 & 34 \\
]250;125] & 34 & 35 & 57 & 41 & 44 & 53 & 69 & 57 & 62 & 58 & 510 \\
]125;90] & 50 & 100 & 23 & 117 & 82 & 125 & 88 & 362 & 167 & 249 & 1363 \\
]90;45] & 43 & 34 & 22 & 61 & 51 & 75 & 54 & 121 & 54 & 32 & 547 \\
Total & 133 & 172 & 102 & 226 & 179 & 255 & 218 & 541 & 286 & 342 & 2454 \\
\hline
\end{tabular}

FP = Forêt Primaire, FS =Forêt secondaire, RPS = Reboisement plurispécifique, TK94 et TKoo plantations forestières industrielles de teck mises en place en 1994 et en 2000. M1 = spores dénombrées après extraction directe M2 = spores dénombrées après piégeage.

FP = Primary forest, FS=Secondary forest, RPS= Multipecific reafforestation, TK94 et TKoo Industrial plantations of Teck initiated in 1994 and 2000. $M 1=$ spore directly counted; $M 2=$ spore obtained using a trap plant.

Tableau 2 : Abondance et densité de spores par type de forêt.

Spore abundance and density according to forest type.

\begin{tabular}{lccccc}
\hline $\begin{array}{l}\text { Taille } \\
\text { des spores }(\mu \mathrm{m})\end{array}$ & FP & FS & RPS & TK $_{94}$ & TKoo \\
\hline Abondance (spores) & 152,5 & 164 & 217 & 379,5 & 314 \\
Densité (sp/g sol) & 0,15 & 0,16 & 0,22 & 0,38 & 0.31 \\
\hline
\end{tabular}

FP $=$ Forêt Primaire, FS = Forêt secondaire, RPS = Reboisement plurispécifique, TK94 et TKoo plantations forestières industrielles de teck mises en place en 1994 et en $2000 . \mathrm{sp} / \mathrm{g}$ de sol $=$ spore $/ \mathrm{g}$ de sol

$F P=$ Primary forest, $F S=$ Secondary forest, RPS = Multipecific reafforestation, TK94 et TKoo Industrial plantations of Teck initiated in 1994 and 2000. sp/g soil = spore $/ \mathrm{g}$ of soil.

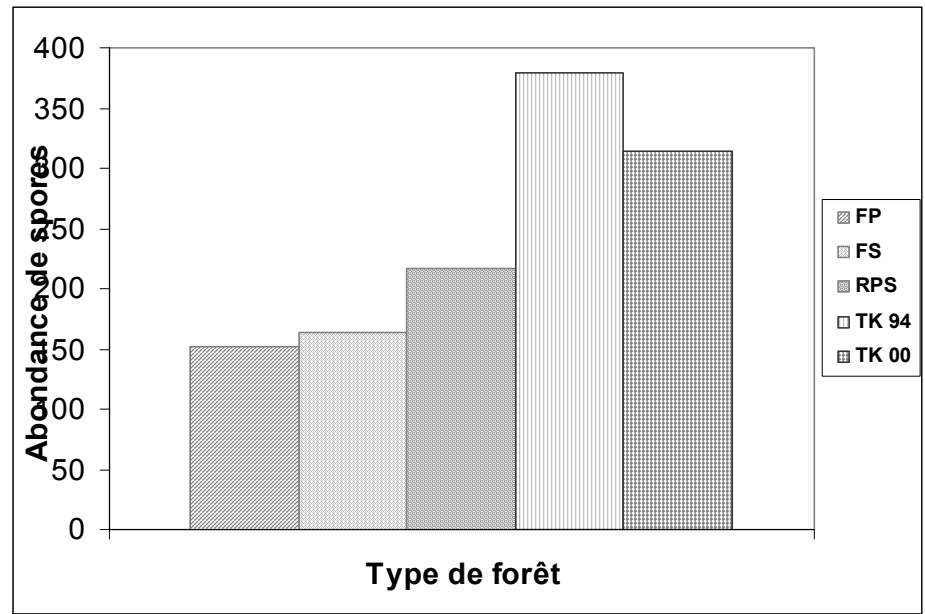

Figure 3 : Abondance des spores dans chaque type de forêt. Spore abundance according to forest type. 


\section{DISCUSSION}

Cette étude montre que les champignons mycorhiziens à arbuscules sont présents dans les différents types de forêts analysées aussi bien dans les plantations forestières industrielles (monospécifiques et plurispécifiques) que dans les forêts naturelles. L'amplification par PCR a permis de révéler que les champignons mycorhiziens au moment de l'échantillonnage étaient déjà présents dans les racines des arbres de la forêt reboisée. La détection moléculaire de champignons mycorhiziens dans les racines de niébé a montré que la technique de piégeage améliore la présence de champignons dans les racines quel que soit le type de sol utilisé comme inoculum. Cela montre que les conditions de culture en pot de la plantepiège pourraient modifier la colonisation racinaire et la sporulation des souches fongiques (Morton, 1993). Ces résultats posent le problème de la durée de vie des souches fongiques (spores) dans le sol avant l'établissement de la symbiose, des facteurs influençant la durée de vie de ces champignons, mais aussi, de la capacité d'une plante à piéger les champignons. Le niébé utilisé est donc une très bonne plante hôte pour la conservation de ces champignons en serre.

II n'a pas été observé de différence significative entre les reboisements monospécifiques et le reboisement plurispécifique. De plus, la différence d'âge des reboisements monospécifiques de Tectona grandis (teck) ne semble pas avoir d'effet significatif sur l'abondance des spores. De même, en forêt naturelle aucune différence significative n'a été observée entre la forêt primaire et la forêt secondaire.

La densité des spores dans le sol est variable ; dans les forêts naturelles, elle est inférieure à la densité des spores dans les forêts issues de reboisement. On pourrait en effet diviser la zone d'étude en deux parties : les Forêts Naturelles (FN) et les Forêts Reboisées (FR), l'abondance étant toutefois plus élevée en FR qu'en FN. Ces résultats indiquent que le type de reboisement (mono ou plurispécifique) et l'âge des reboisements monospécifiques n'ont pas eu, d'effet significatif sur l'abondance des spores. Cette situation pourrait être due au fait que dans les conditions climatiques stables (forêts naturelles), ce sont les réseaux mycéliens qui sont particulièrement impliqués comme source de colonisation mycorhizienne (Jasper et al., 1991) contrairement aux milieux perturbés (forêt issue de reboisement) où les spores jouent un grand rôle (Onguene, 2000). Les zones reboisées ont subi la destruction totale du couvert d'origine. Cette destruction par traitement mécanisée a causé des pertubations profondes dans la structure, la texture et la composition microbiologique du sol. Cette situation pourrait stimuler la sporulation des champignons mycorhizogènes à arbuscules (CMA) dans cette zone par rapport à la forêt naturelle. Cela, en particulier lorsque les éclaircies effectuées dans les plantations forestières industrielles provoquent l'ouverture de la canopée qui entraîne le réchauffement du sol (Smith et Read, 1997).

Dans les plantations forestières industrielles, malgré les différences apparentes entre les types de reboisement (monospécifique ou plurispécifique), l'âge du reboisement, aucune différence du point de vue statistique n'a été observée. Helgason et al. (1999) ont montré qu'après les perturbations, peu de types de spores accroissent leur présence quantitative.

En fonction de la couleur et de la taille, la variabilité observée indique qu'il pourrait exister une diversité taxonomique de Gloméromycètes dans la zone d'étude. Les types de spores observés ne sont pas les mêmes d'une zone à l'autre et d'un point à un autre pour le même type d'utilisation du sol. Cela pourrait s'expliquer par le fait que, les changements dans la composition des hôtes peuvent influencer la composition des champignons associés (Brundett, 1991).

La dispersion des types de spores dans toute la zone d'étude n'est pas homogène. De façon générale, les espèces de CMA ont des cycles de vie très différents et l'abondance relative de chacune d'elles à chaque étape du cycle (spores, hyphes, racines colonisées) peut changer selon le moment où a lieu l'échantillonnage (Brundett et al., 1994).

La majorité des spores est de petite taille car $99 \%$ des spores ont une taille inférieure à $250 \mu \mathrm{m}$ et $78 \%$ une taille inférieure à $125 \mu \mathrm{m}$. II a été montré que les Glomus sont de petite taille (Smith et Read, 1997) et qu'elles sont les plus abondantes en forêt tropicale (Husband et al., 2002). Cela signifie que la plupart des spores extraites pourraient être des Glomus. Cependant, cela reste à confirmer par des analyses taxonomiques plus poussées par la biologie moléculaire (Zézé et al., 1996 ; Helgalson et al., 1999 ; Husband et al., 2002). 


\section{CONCLUSION}

L'étude a permis de mettre en évidence la relative dynamique des populations de champignons de la forêt de la Téné. Elle a montré une différence significative en terme d'abondance de champignons mycorhiziens entre forêts naturelles et forêts reboisées. La détection moléculaire au niveau des racines a permis de façon formelle de mettre en évidence une présence effective de ces champignons en forêts reboisées. Cela est aussi confirmé par la numération des spores tant après comptage direct qu'au piégeage utilisant le niébé. Le niébé est apparu comme un bon hôte pour le développement d'inoculum en laboratoire. $\mathrm{Ce}$ travail est une contribution à la constitution d'une banque de Gloméromycètes en Côte d'Ivoire. II doit se poursuivre afin de permettre de caractériser l'ensemble des CMA inventoriés des sols forestiers, en vue d'une collection locale de ces microorganismes utiles.

\section{REMERCIEMENTS}

Nous sommes reconnaissants aux techniciens du Laboratoire de Phytopathologie de l'Ecole Supérieure d'Agronomie de l'Institut National Polytechnique Houphouet-Boigny, Yamoussoukro (Côte d'Ivoire), pour leur assistance technique au cours de ce travail. $\mathrm{Ce}$ travail a été partiellement financé par le projet Below Ground Biodiversity (GF/2715-02-02$4517,1^{\mathrm{e}}$ tranche).

\section{REFERENCES}

Avenard J. M., M. Eldin, G. Girard, J. Sircoulon, P. Touchebeuf, J. L. Guillaumet, E. Adjanohoun et A. Perraud. 1971. Le milieu naturel de la Côte d'Ivoire. Mém. Orstom, $\mathrm{n}^{\circ} 50,391 \mathrm{p}$.

Barea J. M., C. Azcon-Aguilar and R. Azcon. 1993. Mycorrhiza and crops. Advances in plant pathology $9: 167$ - 189.

Bethlenfalvay G. J. and R. G. Lindermann. 1992. Mycorrhizae in sustainable agriculture. ASA Special Publication $\mathrm{N}^{\circ}$ 54, Madison, Wisconsin, $124 \mathrm{p}$.
Brundett M. C. 1991. Mycorrhiza in natural ecosystems. Adv Ecol Res 21: 71 - 313.

Brundett M. C., L. Melville and R. L Peterson. 1994. Practical methods in mycorrhiza research. Mycological publication, Waterloo, Canada, $161 \mathrm{p}$.

Cissé N., N. N'diaye, S. Thiaw and A. E. Hall. 1995. Registration of «mouride» cowpea. Crop Sci. 35 : 1206 - 1215.

Fitter A. H. 1988. Water relations of red clover Trifolium pratense L. as affected by VA mycorrhizal infection and phosphorus supply before and during drought. Journal of Experimental Botany 39 : 595 - 603 .

Gerdemann and T. H. Nicolson. 1963. Spores of endogone species from soil by wet sieving and decanting. Trans. Br. Myc. Soc. 46 : $235-244$.

Helgason T., A. H. Fitter and J. P. W. Young. 1999. Molecular diversity of arbuscular mycorrhizal fungi colonising Hyacinthoides non-scripta (bluebell) in a seminatural wood land. Molecular ecology $8: 659$ - 666 .

Husband R., E. A Herre and J. P. W. Young. 2002. Temporal variation in the AM communities colonising seedlings in a tropic forest. FEMS Microbiology Ecology 42 : 131 - 136.

Jasper D. A., L. K. Abboth and A. D. Robinson. 1991. The effect of soil disturbance on vesicular-arbuscular mycorrhizal fungi in soil different vegetation types. New Phytol. $118: 471-482$.

Morton J. B. 1993. Problem and solution for the integration of glomalean taxonomy. Mycorrhiza $2: 3$ - 10 .

Onguene N. A. 2000. Diversity and dynamics of mycorrhizal associations in tropical rain forest with different disturbance regime in south Cameroon. Tropenbos-Cameroon Series 3, $167 \mathrm{p}$.

Rogers S. O. and A. J. Bendich. 1985. Extraction of DNA from milligram amounts of fresh herbarium and mummified plant tissues. Plant Mol. Biol. 5 : 69 - 76.

Schüssler A., D. Schawartzzot and C. Walker. 2001. A new phylum, the Glomeromycota : phylogeny and evolution. Mycological Research $105: 1413$ - 1421.

Simon L., Lalonde M. and T. D. Bruns. 1992. Specific amplification of $18 \mathrm{~S}$ fungal ribosomal genes from vesicular-arbuscular endomy- 
corrhizal fungi colonising roots. Applied and Environmental Microbiology 58 : 291 - 295.

Smith S. E., V. Gianinazzi-Pearson, R. Koide and J. W. G. Cairney 1993. Nutrient transport in mycorrhizaes : structure, physiology and consequences for efficiency of the symbiosis. Plant Soil 159: 103 - 113.

Smith S. E. and D. J. Read. 1997. Mycorrhizal symbiosis $2^{\text {nd }}$ edition. Academic Press, San Diego, 605 p.

Trouvelot A., J. L. Kough and V. Gianinazzi-Pearson. 1986. Mesure du taux de mycorhization VA d'un système radiculaire. Recherche des méthodes d'estimation ayant une signification fonctionnelle. In : V. Gianinazzi-
Pearson, S. Gianinazzi (Eds.). The mycorrhizae : physiology and genetics. INRA Presse, Paris, France : pp 217 - 221.

Varma A. 1998. Mycorrhizae, the friendly fungi : what we know, what should we know and how do we know ? In : A. Varma (Eds.). Mycorrhiza manual Springer Berlin. Germany, Heidelberg, New York : pp 1 - 24.

Zézé A., M. Hosny, V. Gianinazzi-Pearson and H. Dulieu. 1996. Characterisation of a highly repeated DNA sequence (SC1) from the arbuscular mycorrhizal fungus Scutellospora castanea and its use as a diagnostic probe in planta. Applied and Environmental Microbiology 62 : 2443 - 2448. 